\title{
Rare Pediatric Phenomenon: Hypoplastic Left Heart Syndrome
}

\section{Rose McGhie-Anderson*}

Department of Nursing, Nova Southeastern University, USA

*Corresponding Author: Rose McGhie-Anderson, Department of Nursing, Nova Southeastern University, USA.

Received: April 12, 2019; Published: May 20, 2019

DOI: 10.31080/ASPE.2019.02.0088

\begin{abstract}
The aim of this review is to heighten the awareness of a critical congenital cardiac disease for the purpose of promoting early diagnosis and intervention especially for those who are primarily at risk. Hypoplastic Left Heart Syndrome (HLHS), is described as hypoplasia of the left ventricle and the ascending aorta, also hypoplasia or atresia of the aortic and mitral valve [1]. HLHS is one of the most complex and challenging congenital heart defects. The left side of the heart is completely compromised and is unable to pump oxygenated blood around the body.

The incidence of HLHS is 1:100,000 live births, which accounts for a total of seven to nine percent of congenital heart diseases. There are approximately sixty seven percent of male infants who are affected with this disorder when compared to females [2].

This paper highlights a real case using a fictitious name to maintain anonymity in presenting the diagnosis, treatment, financial, emotional and the psychological burden this syndrome cause to families, significant others and the healthcare team.

There is a paucity of research available on individuals living with this diagnosis both as children and adults who are experiencing complications and health challenges directly related to HLHS.
\end{abstract}

Keywords: Hypoplastic Left Heart Syndrome; Cardiac; Disease

\section{Introduction}

Kae was diagnosed at 18-20 weeks in utero with a rare congenital heart disease during a routine ultrasound. This diagnosis was confirmed on a fetal echocardiography test as hypoplastic left heart syndrome (HLHS). An early diagnosis of HLHS helps to prepare the parents, families, significant others, and the community of the parents to prepare for this unborn child who has multiple symptoms of left sided heart defects, that has an extremely high risk for morbidity and mortality. This congenital cardiac disease is accompanied with periodic and long-term adjustments to numerous interventions of the multidisciplinary healthcare team especially during the first years of life.

The parents of Kae were young, mother twenty-six and father thirty years old primigravid parents who had strong religious prolife beliefs. Despite recommendations for abortion, they opted to give birth to their new and precious baby girl, whom they named Kae this name means for them, "Sunshine". They have expressed that as parents they believed that God would miraculously heal their unborn child and prayed for this miracle daily. They were carefully optimistic and remarked that even if their hopes were unrealized, their firm belief was that the surgeons could perform interventions that would reverse the cardiac complications they were facing with their infant.

In Baby Kae's case the mitral and aortic valves were completely closed, the aorta and left ventricle were too small, approximately 1.5 millimeters in diameter. The left side of the heart was incapable of supplying blood to the vital organs of the body. Kae had Critical Congenital Heart Defect (CCHD) and unfortunately succumbed to the impact of the defects on her system. CCHD is the most serious form of congenital heart disease (CHD), that requires surgery or some catheter procedure within the first year of life [3]. 
The National Birth Defects Prevention Network (NBDPN) is a group of people who collaborates at the national, state, and local levels to conduct research, track, and prevent birth defects. Their work includes; the assessment of the impact of congenital defects on children, significant others, and the healthcare system. The NBDPN aims at reducing the incidence of other disabilities in children with congenital defects. Various state programs implement vigorous surveillance of congenital heart defects in neonates and children [4]. States that have utilized Critical Congenital Heart Defect surveillance and screening have thirty three percent less of neonatal deaths directly related to CCHD. The principal aim of CCHD is to save the lives of at least 120 more babies annually in the USA [3].

\section{Kae's treatment}

Admitted to the Neonatal Intensive Care Unit immediately post-delivery Kae had a team of expert practitioners, cardiologists, neonatologist and surgeons. She had very little time with her parents and family, this newborn was too sick to accommodate excessive stimulation. She was on a cardiac monitor, she had an arterial line, intravenous catheter, and a nasogastric tube to aid in her treatment to survive [5]. The treatment option proposed to Kae's has three steps to which they signed in agreement an informed consent. Risks and benefits were discussed, along with the possibility of poor outcomes including increased morbidity or mortality.

The first Intervention is a cardiac transplantation, known as the Norwood Procedure, it involves intensive reconstruction and is one of the most challenging pediatric heart surgeries to be done in the first few days of life. Kae was born on March $7^{\text {th }}, 2019$. She was described as alert and responsive to her parents touch and kisses. At birth, intravenous prostaglandin was infused via her umbilical cord vessel to keep the patent ductus arteriosus open. The infusion therapy provided the desired results and the first of a three-part surgery was scheduled for day three, March $10^{\text {th }}, 2019$.

The Norwood Procedure is the first surgical intervention and it involves making the right ventricle as the primary pumping chamber for blood flow to vital organs and around the body. The pulmonary artery and the aorta are also connected closely on either side allowing blood flow that will provide oxygen and nutrients to the cells. Additionally, the aorta will be connected with a BlalockTaussig Shunt, where a tube leading to the lungs is connected to the aorta. A modified Sano Procedure is connected to the right ventricle. Cyanosis is a common symptom for the infant because of the mixing of oxygenated and deoxygenated blood.
The Glenn Shunt was the second procedure planned for Kae. This second procedure is the bidirectional Glenn procedure, typically done at three to six (3-6) months of age This would replace the Blalock-Shunt or Sano Shunt with some other connection to the pulmonary artery and the superior vena cava would be connected to the right pulmonary artery. This will still be mixing the blood and signs and symptoms of cyanosis would persist. The Glenn Shunt is necessary for the preparation of the final procedure.

The Fontan procedure is the final procedure that is performed primarily in children with HLHS, who are older than two or three (2-3) years of age. This surgical procedure allows the following: the mixed blood returning to the heart, the pulmonary artery and the lungs to have more oxygenated blood. The Glenn Shunt remains in place and a second connection is performed and this serves to direct blood from the inferior vena cava to the right pulmonary artery [5].

The psychological impact on family members who lost Baby Kae due to the complications of HLHS are expressed in the following statements:

- Grandmother: "Please pray for my son and his wife they are having bouts of depression".

- Grandfather: "I wish that I could spare them from this experience and go through it for them".

- Aunt: Kae is like a shooting star that quickly brightens your life and is gone so fast but leaves a lasting impact".

- Cousin: "She will never experience the cruelty of this world".

- Kae's Father: "The tears will continue to flow for a very long time".

- Kae's Mother: "There are no words to express my pain right now".

\section{Conclusion}

Substantial inroads have been underway from retrospective research conducted by The National Birth Defects Prevention Study (NBDPS), through surveillance programs in the following states: Arkansas, Georgia, Iowa, Massachusetts, New Jersey, New York, North Carolina, Texas, and Utah. This study supports Kae's case in classifying and diagnosing her congenital cardiac syndrome [6,7]. Currently there is a tremendous need for more surveillance of congenital heart disease among survivors; including growing children and adults and as it relates to the health challenges, health access and usage of care, their overall wellbeing, and the 
determination of strategies that may improve individuals' health overall. The cause of HLHS is unknow and the presumed cause is multifactorial and is purported to be; heredity, which is manifested in an interaction between the genes and environmental factors, or the suggestive notion of an autosomal and recessive dominance. More research is recommended to early diagnose individuals at risk, to obtain early diagnosis of babies in utero and vigilant surveillance of survivors with HLHS diagnosis throughout their life span [2].

\section{Bibliography}

1. Sadineni RT., et al. "Prenatal Sonographic Diagnosis of Hypoplastic Left Heart Syndrome". International Journal of Applied Basic Medical Research 7.3 (2017): 213-215.

2. National Organization for Rare Disorders [NORD]. Hypoplastic Left Heart Syndrome, Rare Disease Database (2007).

3. CDC. CDC'S Tracking and Research for the Prevention of Congenital Heart Defects (2018).

4. Center for Disease Control and Prevention (CDC). Facts about Hypoplastic Left Heart Syndrome (2016).

5. Stanford Children's Health. Hypoplastic Left Heart Syndrome (2019).

6. Reefhuis J., et al. "The National Birth Defects Prevention Study: A review of the methods". National Library of Medicine National Institutes of Health 103.8 (2015): 656-669.

7. Cincinnati Children's. Hypoplastic Left Heart Syndrome/Norwood Procedure (2016).

Volume 2 Issue 6 June 2019

(C) All rights are reserved by Rose McGhie-Anderson.

Citation: Rose McGhie-Anderson. “Rare Pediatric Phenomenon: Hypoplastic Left Heart Syndrome”. Acta Scientific Paediatrics 2.6 (2019): 25 -27. 